\title{
Massive inter-phylum lateral gene transfer from Planctomycetes: the case of TIGR02604 family of the putative glycoside hydrolases
}

\author{
D.G. Naumoff \\ Winogradsky Institute of Microbiology, Research Center of Biotechnology of the Russian Academy \\ of Sciences, Moscow, Russia \\ e-mail:daniil_naumoff@yahoo.com
}

Key words: glycoside hydrolase, $\beta$-galactosidase, TIGR02604, Planctomycetes, Verrucomicrobia, protein evolution, lateral gene transfer, paralogue, orthologue, CAZy

Motivation and Aim: Genome sequencing has revealed that lateral gene transfer is a major evolutionary process in bacteria. Genes of the metabolic enzymes and particularly various glycoside hydrolases are among the most intensively transferred ones. Over 150 glycoside hydrolase families are currently recognized in the Carbohydrate-Active Enzymes database (http://www.cazy.org/). However, a wide diversity of the putative glycoside hydrolases remains unclassified. This is especially true for members of the families which are significantly underrepresented in the well-studied bacterial phyla (Proteobacteria, Firmicutes, and Actinobacteria). One particular example is TIGR02604 family, which is overrepresented in Planctomycetes and Verrucomicrobia (http://www. jcvi.org/cgi-bin/tigrfams/index.cgi). The only experimentally characterized member of this family (GenPept, AGW45552.1) has the $\beta$-galactosidase activity [EC 3.2.1.23]. Detailed phylogenetic analysis of its closest homologues is the purpose of this work.

Methods and Algorithms: Protein sequences were retrieved from the NCBI database. Multiple sequence alignment of TIGR02604-proteins was made in BioEdit program. The phylogenetic trees were built using programs of PHYLIP package.

Results: Eighteen proteins from TIGR02604 family were identified during genome annotation (GenBank, CP019082.1) of the recently described planctomycete from wetlands, Paludisphaera borealis. Pairwise comparison allowed us to group them into ten subfamilies based on the sequence similarity. Four of these proteins belong to the same subfamily as the $\beta$-galactosidase mentioned above. Screening of the database revealed about 200 members of this subfamily and clear overrepresentation of proteins from Planctomycetes. No representatives from Proteobacteria, Firmicutes, Actinobacteria, Cyanobacteria, Chloroflexi, and Spirochaetes were found. Proteins from Acidobacteria, Bacteroidetes, and Verrucomicrobia composed multiple stable clusters on the subfamily phylogenetic tree suggesting massive lateral gene transfer.

Conclusion: Phylogenetic analysis of a new family of putative $\beta$-galactosidases revealed multiple lateral gene transfer events from Planctomycetes to Acidobacteria, Bacteroidetes, and Verrucomicrobia. Poorly studied bacterial phyla are an important source of new enzymes for science and industry.

Acknowledgements: This analysis was performed in frame of the budgetary support (research topic No. 01201350930). 